\title{
Abus et danger du traitement au citrate de clomiphène dans l'infertilité masculine.
}

\author{
M. SCHACHTER, Y. SOFFER \\ Centre d'Infertilité masculine, Service d'obstétrique et de gynécologie. Faculté de Médecine, Uni- \\ versité de Tel Aviv, Tel Aviv, Centre Médical Assaf Harofé, Zérifin, Israël
}

\section{RESUME}

Objectif : Présenter un cas de séminome chez un homme traité au citrate de clomiphène $(\mathrm{CC})$ pour infertilité avec antécédents de cryptorchidie et passer en revue la littérature du séminome dans le contexte des facteurs à haut risque.

Présentation du cas: Il s'agit d'un sujet opéré à 17 ans, d'une double orchidopexie pour cryptorchidie bilatérale. A 29 ans, un traitement au long cours de $\mathrm{CC}$ à la dose de $25 \mathrm{mG} /$ jour lui a été administré pour une infertilité primaire avec oligo-asthéno-tératozoospermie. Quelque amélioration du sperme a été relevée. Divers cycles d'assistance médicale à la procréation durent cependant être mis en ouvre chez le couple. Tous ces efforts furent apparemment couronnés de succès par la survenue, dans l'intervalle des cycles traités, d'une grossesse évolutive heureusement terminée par l'accouchement d'un garçon bien portant. Mais peu de temps après, un séminome typique du testicule droit fut découvert. Trois années de traitement venaient de s'écouler. La littérature relate six cas de tumeurs testiculaires apparues chez des sujets soignés durant des périodes variables pour infertilité par des médications hormonales diverses.

La cryptorchidie est sans aucun doute un facteur à haut risque de malignité. On sait également que les troubles de la spermatogénèse responsables de l'infertilité masculine comportent également des risques non négligeables de malignité. En élevant le taux plasmatique des gonadotrophines et de la testostérone, en activant les récepteurs des œstrogènes ainsi que par d'autres mécanismes moins connus, le citrate de clomiphène est sans aucun doute susceptible de promouvoir des transformations malignes.

En conclusion, la stimulation à long terme des testicules par le citrate de clomiphène requiert beaucoup de prudence, d'autant plus que les avantages de ce traitement sont contestés. Lorsqu'il s'agit, de plus, d'un cas à haut risque en raison de la cryptorchidie, toute stimulation prolongée des gonades devrait être prohibée.

Mots-clés: Gonades, Infertilité masculine, Citrate de Clomiphène, Effets secondaires, Cryptorchidie, Néoplasies testiculaires germinales, Tumeurs malignes.

Correspondance:

Dr Yigal Soffer, Centre d'infertilité masculine, Centre Médical Assaf Harofé IL-70300 Zerifin, Israel Tel: (972) 8-977 9690 Fax: (972) 89779691 email: y.soffer.md@ibm.net $\backslash$ 


\section{INTRODUCTION}

Des travaux récents attirent l'attention sur une relation possible entre la stimulation iatrogène de l'ovulation et les tumeurs malignes de l'ovaire. Cette présomption se fonde sur l'hypothèse du rôle déclenchant de l'extrusion ovocytaire, lors de l'ovulation, sur le développement des tumeurs épithéliales de l'ovaire [4]. Les ovulations incessantes et multiples induites par les gonadotrophines ou le citrate de clomiphène (CC) pourraient donc susciter l'éclosion de tumeurs épithéliales [10, 22). Il faudrait cependant en faire la preuve. L'usage des gonodotrophines dans les infertilités normogonadotropes a toujours été controversé, qu'il s'agisse de gonadotrophines exogènes, de stimulation directe des gonadotrophines endogènes par le $\mathrm{CC}$ ou du rebond de la testostérone. De nombreux travaux montrent que l'augmentation du nombre des spermatozoïdes dans l'éjaculat et du taux des grossesses survenues après administration de CC est discutable. La plupart des cliniciens s'opposent aux traitements empiriques n'ayant pas fait leurs preuves dans des études bien contrôlées. Les partisans de ces thérapeutiques soulignent néanmoins la rareté et la modération de leurs effets secondaires.

Nous rapportons un cas de séminome testiculaire apparu 16 ans après orchidopexie pour cryptorchidie et à la suite de 3 années de traitement au CC.

\section{PRÉSENTATION DU CAS}

M. H.Y..., âgé de 31 ans, d'origine juive nordafricaine était en traitement dans un autre centre d'infertilité où il était suivi régulièrement. Une orchidopexie pour cryptorchidie bilatérale a été pratiquée à l'âge de 17 ans. Il a été vu chez nous lorsque sa femme a été admise pour traitement dans notre centre infertilité féminine et de FIV. Il prenait depuis plus d'un an $25 \mathrm{mG} /$ jour de CC. A l'examen physique, les caractères sexuels secondaires étaient normaux. Les testicules étaient bien développés, $22 \mathrm{~mL}$ chacun, et de consistance normale. La verge, le méat urétral, les canaux déférents et les épididymes étaient également normaux. L'analyse du sperme montrait les résultats suivants: volume, $2,8 \mathrm{~mL}, \mathrm{pH} 8,1$; taux de fructose normal. Numération des spermatozoïdes, $14 \times 106 / \mathrm{mL}$; vitalité $85 \%$; mobilité $35 \%$ (degré II, non progressive); formes normales $9 \%$ (critères stricts). Test du SpermMar $8 \%$ de spermatozoïdes attachés. Test de pénétration du hamster 15\% (normal 20-100\%). Spermoculture (chlamydia, mycoplasmes, germes banaux) négative. Taux hormonaux (après arrêt du CC pour deux semaines): FSH 2,2 $\mathrm{mIU} / \mathrm{mL}$, LH 5,2 $\mathrm{mIU} / \mathrm{mL}$, Prolactine $10,4 \mathrm{nG} / \mathrm{mL}$, et testostérone $13.4 \mathrm{nmol} / \mathrm{ml}$.

Chez la femme, le test post-coital était mauvais mais l'ovulation était normale, ainsi que l'utérus et les trompes à l'hystérosalpingographie. Cinq cycles d'inséminations intra-utérines furent pratiqués ainsi que deux cycles de FIV avec micro-injection (ICSI). L'examen de sperme fut répété chez nous, pour contrôle et ne montra pas de changement sensible: Volume, 4 $\mathrm{mL}, \mathrm{pH}, 7,9$, vitalité $80 \%$, compte $18 \times 106 / \mathrm{mL}$, mobilité $33 \%$, forme normale $10 \%$. Pendant tout ce temps, M. H.Y... continuait à prendre du CC comme prescrit. Au premier cycle de FIV/ICSI deux ovocytes sur 23 étaient fécondés, sans grossesse. Au second cycle, neuf ovocytes sur 32 étaient fécondés. La femme devint enceinte, mais avorta à la 9ème semaine.

Deux mois après, la femme redevint spontanément enceinte et donna naissance, à terme, à un petit garçon bien portant. Peu de temps après l'accouchement, M. H.Y... remarqua la présence d'un nodule grandissant dans le pôle supérieur du testicule droit. L'examen révéla un nodule régulier et dur de $4 \times 3 \mathrm{~cm}$ distinct des tissus environnants, un cordon spermatique normal, et l'absence d'adénopathies inguinales. Une orchidectomie radicale droite fut pratiquée. L'examen histo-pathologique témoignait d'un séminome typique sans métastases apparentes. Au total, l'administration de $\mathrm{CC}$ se prolongea trois années.

\section{DISCUSSION}

Le séminome est la tumeur testiculaire d'origine germinale la plus commune. Elle est de 2,5 à 20 fois plus fréquente chez les sujets cryptorchides que chez les hommes normaux 
$[3,5,6]$. Il semble que cela soit vrai même aprés une orchidopexie avec l'abaissement des testicules à leur place dans le scrotum. Pour limiter les dégâts infligés à l'épithélium séminifère et réduire les risques de transformation maligne, la plupart des auteurs recommandent d'effectuer la descente des testicules vers l'âge de six ans au plus tard par quelque méthode que se soit, hormonale ou chirurgicale. Tout retard dans la mise en ouvre du traitement médical ou chirurgical accroît les risques de malignité (Einhorn et al, 1993). Le fait que des cellules cancéreuses in-situ aient été observées dans des testicules cryptorchides chez des garçons pré-pubères [20], montre que cette malignité peut se développer dans l'enfance. Le développement de la tumeur est cependant contenu jusqu'à la puberté, mais il n'en est plus de même lorsque le taux des gonadotrophines s'élève. Des aspects caractéristiques de cellules germinales atypiques ont été décrits dans les testicules chez des sujets cryptorchides et/ou infertiles ainsi que dans le testicule contro-latéral sain chez des sujets porteurs d'un cancer germinal du testicule $[7,8,15,19]$. Les sujets présentant ces anomalies cytologiques sont exposés à un risque accru de tumeurs germinales invasives du testicule. Dans le cas présent, la correction chirurgicale de la cryptorchidie a été faite tard, à l'âge de 17 ans, exposant notre sujet à un risque accru et double d'infertilité et de malignité. Dans un groupe de 650 sujets traités au CC pour infertilité, [13] deux cas de tumeurs germinales furent découverts. Chez ces deux sujets, le bilan endocrinien témoignait d'une bonne réponse au CC et l'analyse du sperme montrait, à la suite de ce traitement, une élévation sensible du compte des spermatozoïdes. Ces sujets recevaient du CC trois semaines sur quatre durant six à douze mois. Les cas de cryptorchidie étaient exclus de cette étude en raison de la triade bien connue de cryptorchidie, d'infertilité et de malignité [13]. Quatre autres cas de tumeurs associées à un traitement hormonal ont été décrits:

1) Le cas d'un séminome testiculaire découvert chez un homme de 27 ans [12]. Il avait reçu d'une manière continue pendant 15 mois, pour infertilité primaire et oligo-asthe- nozoospermie, $50 \mathrm{mG} / \mathrm{jour}$ de $\mathrm{CC}$ et 100 $\mathrm{mG} /$ jour de mestérolone.

2) le cas d'une tumeur combinant tératome et séminome atypique chez un homme de 28 ans [17] ayant reçu du cis-clomiphène à la dose de $1 \mathrm{mg} /$ jour pendant 21 semaines.

3) le cas d'un tératome testiculaire chez un homme de 34 ans [18] ayant reçu des gonadotrophines ménopausales humaines à la dose de $225 \mathrm{IU} /$ semaine pendant 10 semaines ainsi que des gonadotrophines chorioniques humaines à la dose de $1500 \mathrm{IU} /$ semaine pendant 8 semaines.

4) Le cas d'une tumeur des cellules de Leydig chez un homme de 45 ans [1] avec des antécédents de cryptorchidie traitée dans son enfance, ayant reçu pendant trois mois seulement un traitement intermittent ( 15 jours / mois) au CC à la dose de $50 \mathrm{mG} /$ jour. Dans tous ces cas, le rôle possible du traitement a été relevé.

Quant à la relation de cause à effet entre la cryptorchidie, le séminone et l'infertilité, elle est loin d'être claire. La température plus élevée dans la cavité pelvienne que dans le scrotum pourrait à la longue provoquer dans le testicule un dérèglement de la différenciation et de la prolifération du tissu germinatif. Les cellules germinales de l'ovaire, entrées toutes en phase II de méiose à la naissance, seraient moins exposées à ce risque. Il se pourrait également que la cryptochidie soit due à quelque défaut génétique des cellules germinales. Ce défaut serait par la suite, à l'âge adulte, responsable du risque accru d'infertilité et de malignité. En faveur de cette théorie génétique, plaide l'éclosion à caractère familial de ces tumeurs testiculaires [11], ainsi que leur association fréquente à de nombreux autres néoplasmes [2]. Les tumeurs malignes du testicule ont également été liées à l'infertilité dans trois séries totalisant 2850 hommes infertiles. Onze tumeurs malignes du testicule ont été découvertes $(0,38 \%)$ chez ces hommes, alors que leur fréquence n'est que de $0,003 \%$ dans la population générale $[9,14,19]$. Une étude anglaise bien contrôlée, [21] faite sur 794 hommes présentant des tumeurs et sur des contrôles 
appariés, a mis en évidence un lien associatif entre les tumeurs malignes du testicule d'une part et la cryptorchidie, la hernie inguinale, la puberté précoce et l'infertilité d'autre part.

Dans l'infertilité masculine, les traitements hormonaux aux gonadotrophines ou au CC sont administrés dans le but d'intensifier, directement ou indirectement, la spermatogenèse et la sécrétion des androgènes. Admettant que ce mécanisme puisse induire une spermatogénèse forcée, à la manière de l'ovulation incessante chez la femme, il pourrait avoir aussi un effet oncogène sur la lignée germinale mâle. Dans une revue exhaustive, [16], le rôle possible des hormones dans le développement du cancer du testicule est longuement débattu. Les ostrogènes seraient impliqués, durant la période prénatale, à la puberté et à l'âge adulte, dans la pathogénèse de la cryptorchidie, des testicules hypoplasiques et des anomalies du sperme. De nombreux produits à activité œstrogénique sont largement répandus dans notre environnement; on leur impute la recrudescence récente du cancer testiculaire. Le CC avec ses propriétés œstrogéniques et anti-œstrogéniques, pourrait exercer un effet carcinogène direct sur la lignée germinale ou avoir une action indirecte par l'entremise des cellules de Sertoli et de Leydig. L'exposition aux œstrogènes pourrait déprimer le facteur inhibiteur antimullérien (MIF), rompre l'équilibre hormonal et aboutir à une élévation incontrôlée du taux des gonadotrophines. Des taux élevés de gonadotrophines ont été aussi impliqués dans la pathogénèse des cancers du testicule à cause de leur effet stimulant sur la division méiotique des cellules germinales. L'éclosion de tumeurs germinales survient ou succède à des périodes où les gonadotrophines sont élevées, telle que la période néo-natale pour les tumeurs du sac allantoïdien ou la période post-pubertaire pour les séminomes. Le CC, en stimulant la sécrétion des gonadotrophines, pourrait agir par leur influence ou par quelque synergisme avec les facteurs de croissance présents dans les tubes séminifères. Les états hypogonadotropes sont rarement associés aux tumeurs testiculaires. En dépit de cryptorchidies fréquentes, les por- teurs d'un syndrome de Kallman ne développent pas de tumeurs testiculaires. Les hommes dont les testicules sont hypotrophiés et les cellules de Sertoli peu nombreuses ont des taux de gonadotrophines généralement élevés. Aussi, seraient-ils particulièrement vulnérables à la dégénerescence maligne de cellules germinales normales ou atypiques et au développement de foyers cancéreux insitu. Un autre effet des gonadotrophines est l'élevation des androgènes. Des taux élevés de testostérone et de dihydrotestostérone, consécutifs à la stimulation des cellules intestitielles, peuvent avoir un effet nocif sur l'activation et la différenciation des cellules germinales mâles.

Pour revenir à notre patient, se serait pure spéculation conjoncturelle d'associer le séminome au CC sur la base de notre seul cas, connaissant ses antécédents de cryptorchidie bilatérale tardivement corrigée. Cette association n'est rapportée que très sporadiquement jusqu'à présent chez des hommes infertiles sans cryptorchidie, en dépit du grand nombre de cas ainsi traités. Dans le seul cas de la littérature [1] où il y avait aussi des antécédents de cryptorchidie, le traitement intermittent au CC, voir ci-haut, ne s'était pas prolongé au délà de 3 mois, jettant, de l'avis même des auteurs, des doutes sur la relation de cause à effet entre l'instauration du traitement et l'éclosion de la tumeur leydigienne. Notre patient recevait d'une manière continue pendant trois ans du CC à la dose de $25 \mathrm{mG}$ /jour. D'autres rapportent des doses continues variant de $50 \mathrm{mG} /$ jour pendant 15 mois à $25 \mathrm{mG} /$ jour pendant 6 mois. Néanmoins, se serait manquer de bon sens que de méconnaître cette association dans notre cas et d'affirmer que le CC n'y est pas impliqué. Dans un effort de dépistage précoce du séminome et d'autres tumeurs testiculaires, les sujets opérés d'orchidopexie doivent être soigneusement suivis, même après de longues années. De plus, les médications dont l'efficacité et les dangers ne sont pas élucidés d'une manière indubitable devraient être prescrits avec prudence, surtout chez des sujets à haut risque. 


\section{REFERENCES}

1. BUJAN L., MIEUSET R., DE ZONG Z., MANSAT A., PONTONNIER F.: Development of Leydig cell tumour in association with clomiphene treatment for oligozoospermia. Brit J Urol, 1992; 69 (6): 659-60.

2. DIECKMAN K.P., WEGNER H.E., KRAIN J. Multiple primary neoplasms in patients with testicular germ cell tumors. Oncology, 1994; 51: 450-8.

3. EINHORN L.H., RICHIE J.P., SHIPLEY W.U.: Cancer of the testis. In: De Vita VT., Helman S. and Rosenberg SA, eds. Cancer: Principles and Practice of Oncology 4th Ed. Philadelphia J.B. Lippincott Co, 1993: 1126-46.

4. FATHALLA M.F.: Incessant ovulation- a factor in ovarian neoplasia [lettre] Lancet, 1971;2: 163.

5. FARRER J.H. WALKER A.H., RAJFER J.: Management of the postpubertal cryptorchid testis: A statistical review, J Urol, 1985; 134: 1071-5.

6. FONGER J.D., FILLER R.M., RIDER W.D.: Testicular tumors in maldescended testes. Can J Surg, 1981; 24: 353-5.

7. GIWERCMAN A., MARKS A., SKAKKEBEACK N.E.: Carcinoma-in-situ germ cells exfoliated from seminiferous epithelium into seminal fluid (lettre) Lancet 1988; i:530.

8. GIWERCMAN A., CLAUSEN O.P.F., SKAKKEBEACK N.E.: Carcinomi-in-situ in the testis: aneuploid cells in semen. Brit Med $J$; 1988; 296: 1762-4.

9. GRIFFIN J.E. AND WILSON J.D.: Disorders of the testes and the male reproductive tract, In: Williams Textbook of Endocrinology, 8th ed. Wilson J.D. and Foster D.W. eds. pp.799-852. W.B. Saunders, Philadelphia PA.

10. HARTGE P., SCHIFFMAN M.H., HOOVER R., MCGOWAN L., LESHER L. AND NORRIS HJ.: A case control study of epithelial ovarian cancer. Am J Obstet Gynecol, 1989; 161: 10-16.

11. MOLLER H.: Clues to the etiology of testicular germ cell tumors from descriptive epidemiology. Eur Urol, 1993; 23(1): 8-13.

12. NEOPTOLEMOS J.P., LOCKE T.J., FOSSARD D.P. Testicular tumorr associated with hormonal treatment for oligospermia. Lancet ,1981; ii: 754 .

13. NILSSON A., AND NILSSON S.: Testicular germ cell tumors after clomiphene therapy for subfertility. J Urol, 1985; 134: 560-1.

14. NUESCH-BACHMANN I.H.R., HEDINGER C.: Atypische spermatogonien als prae kanzerosc. Schweiz Med Wochenschr, 1977; 107: 795-801.

15. PARKINSON M.C., SWERDLOW A.J., PIKE M.C.: Carcinoma-in-situ in boys with cryptochordism: When can it be detected? Brit J Urol, 1994; 73:431-5.
16. RAJPERT-DE MEYTS E., SKAKKEBEAK N.E.: The possible role of sex hormones in the development of testicular cancer. Eur Urol, 1993; 23: 54-61.

17. REYES F.J., FAIMAN C.: Developnaent of a testicular tumor during cisclomiphene therapy. Can Med Assoc J, 1973; 109; 502-6. 15.

18. RUBIN S.O.: Malignant teratoma of testis in a subfertile man treated with HCG and HMG. Scand J Urol Nephol, 1973; 7: 81-4.

19. SKAKKEBEAK N.E.: Carcinoma in situ of the testis: Frequency and relationship to invasive germ cell tumors in infertile men. Histopathol, 1978; 2: 152-70.

20. SKAKKEBEAK N.E., BERTHELSEN J.G.: Carcinoma-in-situ and development of different types of germ cell tumors. Fortsch Androl, 1981: 7: 89-90.

21. United Kingdorm Testicular Cancer Study Group, Etiology of Testicular Cancer: Association with congenital abnormalities, age at puberty, infertility and exercise. Brit Med J, 1994; 38: 1393-9.

22. WHITTEMORE A.S., HARRIS R., INTYRE J., and the Collaborative Ovarian Cancer Group. Characteristics relating to ovarian cancer risk; collaborative analysis of 12 US case-control studies IV. The pathogenesis of epithetelial ovarian cancer. J Epidemiol, $1992 ; 136 ; 1212-20$.

\section{ABSTRACT \\ Clomiphene citrate treatment abuse and risks in male infertility \\ M. SCHAChTER \& Y. SOFFER \\ Obiective: To describe a case of seminoma occurring in a patient with a past history of cryptorchidism, infertility and clomiphene citrate (CC) treatment. To review the literatu- re of seminoma in association with these risk} factors.

Case report: A 33 year old man developed typical seminoma in the right testis. A bilateral cryptorchidism was lately diagnosed and treated by orchidopexy at the age of 17. Primary infertility due to oligoasthenospermia was diagnosed at the age of 29 . Treatment was initiated with $\mathrm{CC}$ at the dose of 25 mG/day. Semen quality improved gradually. After failure of various assisted reproductive techniques, a spontaneous pregnancy was achieved and a healthy baby delivered. Shortly after delivery the seminoma was diagnosed, after a total of three years of treatment. Six cases of testicular tumors have been reported in the literature in which 
patients had been pretreated with fertility drugs for various periods of time before tumors was diagnosed. Cryptorchidism is a well-known risk factor for testicular malignancy, as is the infertile state. Clomiphene citrate may promote malignant transformation by activation of estrogen receptors, by raising gonadotropin and/or testosterone concentrations, or by other mechanisms.

Conclusion: Clomiphene citrate may have a previously unappreciated malignancy-promoting effect in infertile men, especially those with other risk factors. Clinicians treating similar patients should take this into account and the literature monitored for other associated cases.

Key-words: Germ Cell Tumor, Malignancy Gonads, Clomiphene Citrate, Drug- Adverse effects, Infertility - Male, Cryptorchidism. 